\title{
Yellow nails, lymphedema and chronic cough: Yellow nail syndrome in an eight-year-old girl
}

\author{
Ishita Siddiq MSc ${ }^{1}$, Daniel Hughes MD FRCPC ${ }^{2}$
}

\begin{abstract}
I Siddiq, D Hughes. Yellow nails, lymphedema and chronic cough: Yellow nail syndrome in an eight-year-old girl. Can Respir J 2012;19(1):35-36.
\end{abstract}

Yellow nail syndrome is a rare disease and reported mainly in adults. A case of yellow nail syndrome involving an eight-year-old girl with associated discoloured yellowish nails on the fingers and toes, lymphedema and chronic cough, and sputum production is reported.

Key Words: Chronic cough; Keratosis obturans; Lymphedema; Yellow nail syndrome

First described in 1964 (1), yellow nail syndrome (YNS) was primarFily diagnosed by the appearance of yellow nails and lymphedema. It was later noted that YNS was associated with a variety of respiratory disorders and a triad for diagnosis was formed (2). Respiratory illnesses observed included pleural effusion in 35\% to $40 \%$ (3-5), bronchiectasis in 20\% (5-7) and sinusitis in $83 \%(8,9)$ of patients. The diagnosis of YNS should be considered in a patient with two of the three findings associated with the disorder. Published cases have differed in their manifestations. One case involving a 40-year-old woman noted keratosis obturans (the accumulation of extra skin cells and wax in the external auditory canal) to be associated with YNS, thereby adding complications to the external ear as a possible sign of the disorder (10). To date, there have been approximately 150 cases reported in the literature (11). Although the pathogenesis of YNS is unclear, it is believed that the syndrome may be due to a structural and functional dysfunction of the lymphatic system coupled with an increased vascular permeability to albumin resulting in the observed lymphedema and yellow nails (12). The respiratory illness component is believed to be due to hypoplasia of the lymphatic system or an immunological deficiency (13). Causes of YNS remain unknown; however, some suspect that it occurs secondary to conditions such as connective tissue disease, thyroid disorder or, in some cases, an adverse effect to drugs such as penicillamine and bucillamine (13). Cases of YNS have been typically reported in patients between 40 and 60 years of age, and the observation of YNS in the pediatric population is extremely rare $(14,15)$. The present case report describes an eight-year-old girl who exhibited the classic triad of YNS.

\section{CASE PRESENTATION}

An eight-year-old girl was referred to the Chest Clinic at the IWK Health Centre (Halifax, Nova Scotia) with a history of chronic cough since one year of age. Following a normal 41-week pregnancy, she was born weighing $3.77 \mathrm{~kg}$. At birth, she suffered a left-sided pneumothorax and pneumomediastinum not requiring drainage. Physical examination demonstrated slight dysmorphic features including a flat philtrum and thin lips. The most noticeable abnormality was the absence of nails on her fingers and toes. Genetic consultation was sought; karyotyping revealed 46 normal XX chromosomes, but no specific genetic diagnosis was made. By one year of age, she developed a chronic loose cough, eventually productive of greenish yellow sputum and discomfort in her right ear. A pediatric otolaryngologist noted mild soft tissue thickening, and irregularity of the right tympanic membrane and external auditory canal. She developed a

\section{Les ongles jaunes, le lymphœdème et la toux chronique : le syndrome des ongles jaunes chez une fillette de huit ans}

Le syndrome des ongles jaunes est une maladie rare déclarée surtout chez des adultes. Les auteurs présentent un cas de syndrome des ongles jaunes chez une fillette de huit ans présentant des ongles de doigts et d'orteils jaunes et décolorés, un lymphœè̀me, une toux chronique et une production d'expectorations.

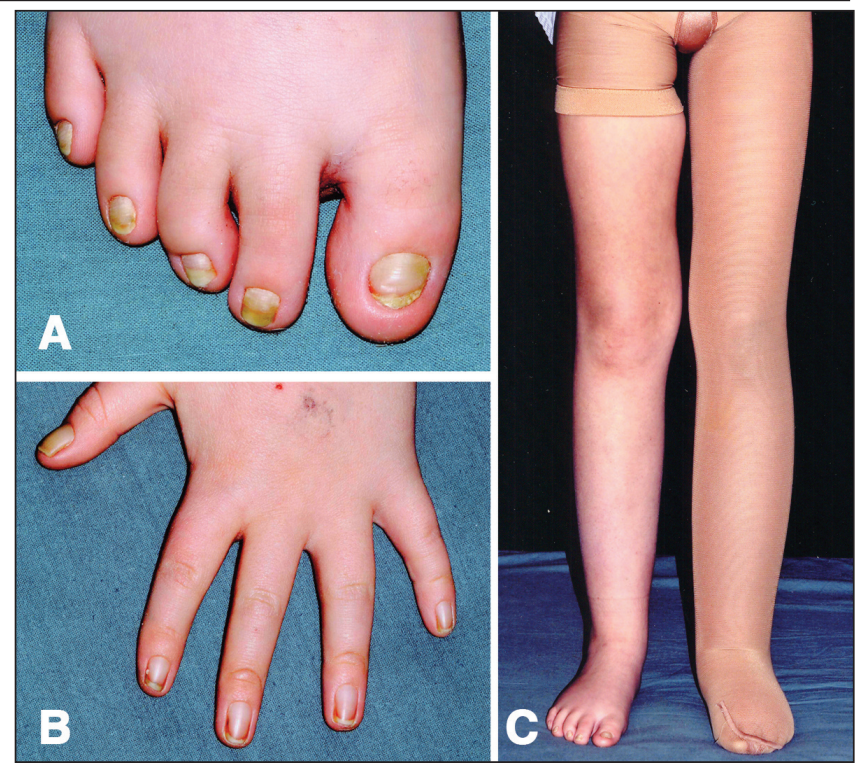

Figure 1) The toes (A) and fingernails (B) of an eight-year-old girl demonstrating the yellow-coloured, thick and transverse ridged features of nails in yellow nail syndrome. The compression stockings (C) worn on her left leg to manage the lymphedema

chronic right ear discharge, and granulation in the right ear canal at the junction of the cartilaginous and bony portions of the canal. An otowick was placed in her right ear and her chronic ear infections were treated with oral antibiotics. While on antibiotics, an improvement in her cough was noted. At the end of her antibiotic treatment, however, her cough and ear discomfort recurred. A computed tomography scan ruled out middle ear disease and cholesteatoma as causes of the discomfort. At two years of age, she was diagnosed with leftsided lymphedema involving her arm and leg. The lymphedema improved with age, becoming more localized to her left leg. Nail growth gradually appeared; however, they appeared discoloured with transverse ridges and grew very slowly (Figures $1 \mathrm{~A}$ and B). She was treated with a compression stocking to increase lymph flow from her affected leg (Figure 1C).

Current physical examination showed a normally grown girl: height $133 \mathrm{~cm}$ (50th to 75th percentile) and weight $28.6 \mathrm{~kg}$ (50th percentile),

${ }^{1}$ Faculty of Medicine, Dalhousie University; ${ }^{2}$ IWK Health Centre, Halifax, Nova Scotia

Correspondence and reprints: Dr Daniel Hughes, IWK Health Centre, 5850/5980 University Avenue, Halifax, Nova Scotia B3K 6R8.

Telephone 902-470-8218, fax 902-470-7223, e-mail dan.hughes@iwk.nshealth.ca 


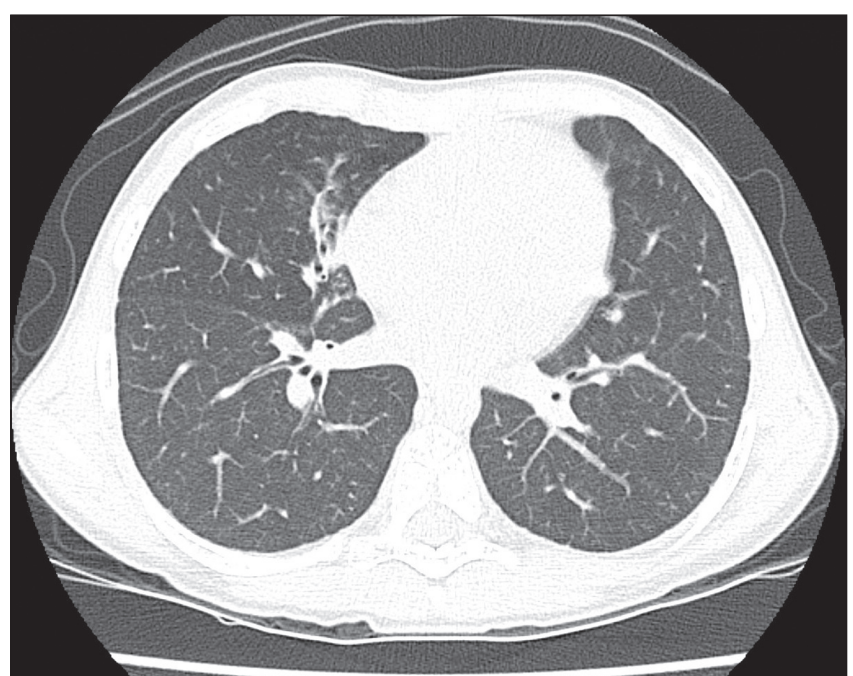

Figure 2) A single-slice chest computed tomography scan demonstrating mild bronchial dilation

with the nail and lymphedema findings as shown in Figure 1. She had some granulation in her left ear canal. She was not acutely distressed but had a loose cough productive of whitish sputum. Breath sounds were symmetric, and no crepitations or wheezes were heard. There were no chest wall abnormalities or finger clubbing. Laboratory investigations included complete blood count, immunoglobulins and sweat chloride, which were normal. Her serum albumin level was normal $(46.4 \mathrm{~g} / \mathrm{L})$. Her sputum grew Haemophilus influenza and Streptococcus pneumonia, and she was placed on oral antibiotics. Subsequent sputum cultures have grown H influenza. Pulmonary function testing showed a normal forced vital capacity (FVC) of $2.19 \mathrm{~L}$ (111\% predicted), forced expiratory volume in $1 \mathrm{~s}\left(\mathrm{FEV}_{1}\right)$ of $1.33 \mathrm{~L}$ ( $78 \%$ predicted), a reduced $\mathrm{FEV}_{1} / \mathrm{FVC}$ ratio of 0.61 and a reduced forced expiratory flow between $25 \%$ and $75 \%$ of FVC of $0.76 \mathrm{~L} / \mathrm{s}$ (36\% predicted), with no significant response to bronchodilator. A chest $\mathrm{x}$-ray demonstrated mild hyperinflation and central peribronchial thickening and mild subsegmental atelectasis of the right middle lobe. There was no evidence of pleural effusion. A chest computed tomography scan revealed mild bronchial wall thickening in the right lung, with mild dilation of some third-and fourth-generation bronchi in the right middle lobe, but no definite radiographic evidence of bronchiectasis (Figure 2).

\section{DISCUSSION}

The details of the patient's history and findings suggest that she was one of the few cases of YNS seen in the pediatric population. She exhibited three characteristics that comprise the YNS triad: yellow nails, lymphedema and a chronic cough producing sputum. Armitage et al (10) described a patient presenting with keratosis obturans in addition to all three hallmarks of YNS. This finding may explain the ear difficulties noted in our patient. Early detection of YNS in our patient will enable proper treatment of her chronic cough and potentially prevent irreversible damage to her lungs such as bronchiectasis.

The uncertainty over the cause and pathogenesis of YNS makes it difficult to treat. Primarily considered to be a syndrome that affects the adult population, YNS is more recently emerging in the pediatric population. The present case represents one of a handful of cases noted in children. Case studies of adult patients with bronchiectasis report some benefits from postural drainage and antimicrobial therapy. Therapeutic thoracenteses were used to treat pleural effusions, while some underwent pleurodesis for the management of relapses. Patients suffering from symptomatic chylothorax had the option of undergoing a thoracic duct ligation (16) and were encouraged to eat a low-fat diet (17). Lymphedema in the patients did not clear. However, improvements have been noted in patients on decongestive therapy (18) and those wearing compression bandages. Management of the respiratory and lymphatic drainage problems was associated with an improvement in the nail manifestations without specific, nail-targeted treatment (17). This observation encouraged some physicians to use the nail discolouration as a 'barometer' of the syndrome. Some physicians have used vitamin $\mathrm{E}$ therapy and topical steroids without any clear evidence of improvement (18). The prognosis of YNS is uncertain. A series of case studies have suggested that the life expectancy of a YNS patient is moderately reduced compared with the general population (17). The disruption to the lymphatic system remains the most likely cause of YNS. The clinical manifestations can be treated individually and, if managed appropriately, the long-term outcome should be satisfactory.

In addition to compression stockings, our patient is currently treated with regular bronchodilator aerosols and chest physiotherapy (mainly a positive expiratory pressure mask) along with oral antibiotic courses during respiratory exacerbations associated with viral respiratory infections. Our patient has chronic cough and sputum production, but no radiographic evidence of bronchiectasis. We hope to prevent the onset of bronchiectasis, which has been associated with this disorder. Further genetic testing is underway. She has been fully immunized, except for the new Prevnar 13 (Wyeth, Canada), which has been recommended.

The present case report alerts physicians to the existence of YNS in the pediatric population and to its association with the development of bronchiectasis. Early management using airway clearance techniques and antibiotics may prevent irreversible damage to the lungs.

\section{REFERENCES}

1. Samman PD, White WF. The "yellow nail syndrome". Br J Dermatol 1964;76:153-7.

2. Alkadhi H, Wildermuth S, Russi EW, Boehm T. Yellow nail syndrome. Respiration 2005;72:197.

3. Biscetti F, Straface G, DeAngelis G, Flex A. Yellow nails, ankle edema, and pleural effusion. Cleve Clin J Med 2009;76:317-8.

4. Brofman JD, Hall JB, Scott W, Little AG. Yellow nails, lymphedema and pleural effusion. Treatment of chronic pleural effusion with pleuroperitoneal shunting. Chest 1990;97:743-5.

5. Rubin B. Overview of cystis fibrosis and non-CF bronchiectasis. Semin Respir Crit Care Med 2003;24:619-28.

6. Bowers D. Unequal breasts, yellow nails, bronchiectasis and lymphedema. CMAJ 1969;100:437-8.

7. McNicholas WT, Quigley C, FitzGerald MX. Upper lobe bronchiectasis in the yellow nail syndrome: Report of a case. Ir J Med Sci 1984;153:394-5.

8. Elmariah, SB, Ubriani RR, Kovich O. Yellow nail syndrome. Dermatol Online J 2008;14:17.

9. Varney VA, Cumberworth V, Sudderick R, Durham SR, Mackay IS. Rhinitis, sinusitis and the yellow nail syndrome: A review of symptoms and response to treatment in 17 patients. Clin Otolaryngol Allied Sci 1994;19:237-40.

10. Armitage JM, Lane DJ, Stradling JR, Burton M. Ear involvement in the yellow nail syndrome. Chest 1990;98:1534-5.

11. Maldonado F, Ryu JH. Yellow nail syndrome. Curr Opin Pulm Med 2009;15:371-5.

12. D’Alessandro A, Muzi G, Monaco A, Filiberto S, Barboni A, Abbritti G. Yellow nail syndrome: Does protein leakage play a role? Eur Respir J 2001;17:149-52.

13. Nanda S, Dorville F. Yellow nail syndrome. CMAJ 2009;181:614

14. Cebeci F, Celebi M, Onsun N. Nonclassical yellow nail syndrome in six-year-old girl: A case report. Cases J 2009;2:165-7.

15. Paradisis M, Van Asperen P. Yellow nail syndrome in infancy. J Paediatr Child Health 1997;33:454-7.

16. Zarogiannis S, Hatzoglou C, Molyvdas PA, Gourgoulianis K. Yellow nail syndrome chylous pleural effusions. Defective lymph valves involved? Chest 2008;134:1353.

17. Maldonado F, Tazelaar HD, Wang C, Ryu JH. Yellow nail syndrome: Analysis of 41 consecutive patients. Chest 2008;134:375-81.

18. Szolnoky G, Lakatos B, Husz S, Dobozy A. Improvement in lymphatic function and partial resolution of nails after complex decongestive physiotherapy in yellow nail syndrome. Int J Dermatol 2005;44:501-3. 


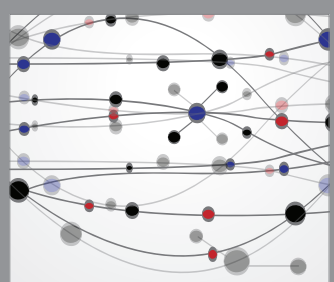

The Scientific World Journal
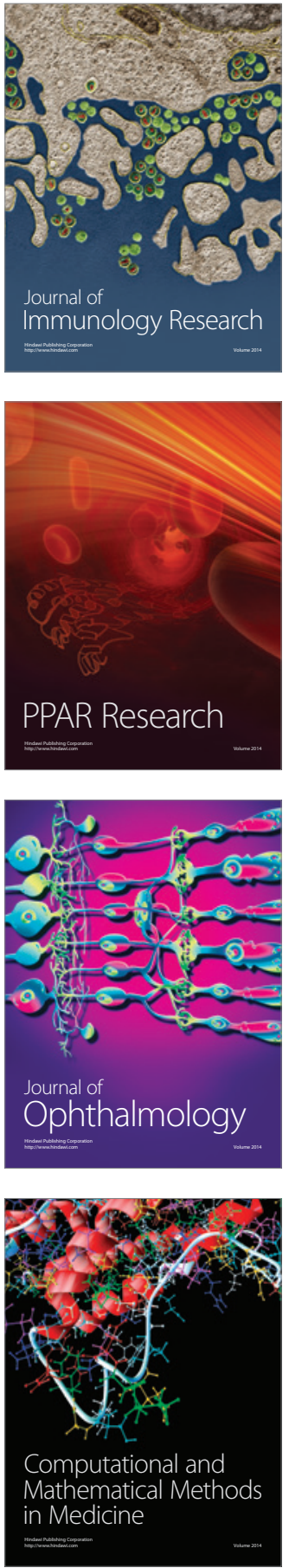

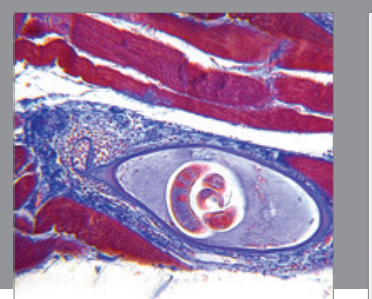

Gastroenterology Research and Practice

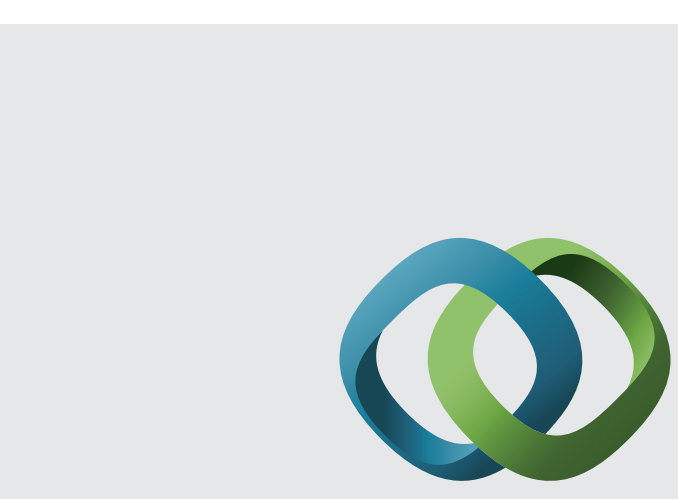

\section{Hindawi}

Submit your manuscripts at

http://www.hindawi.com
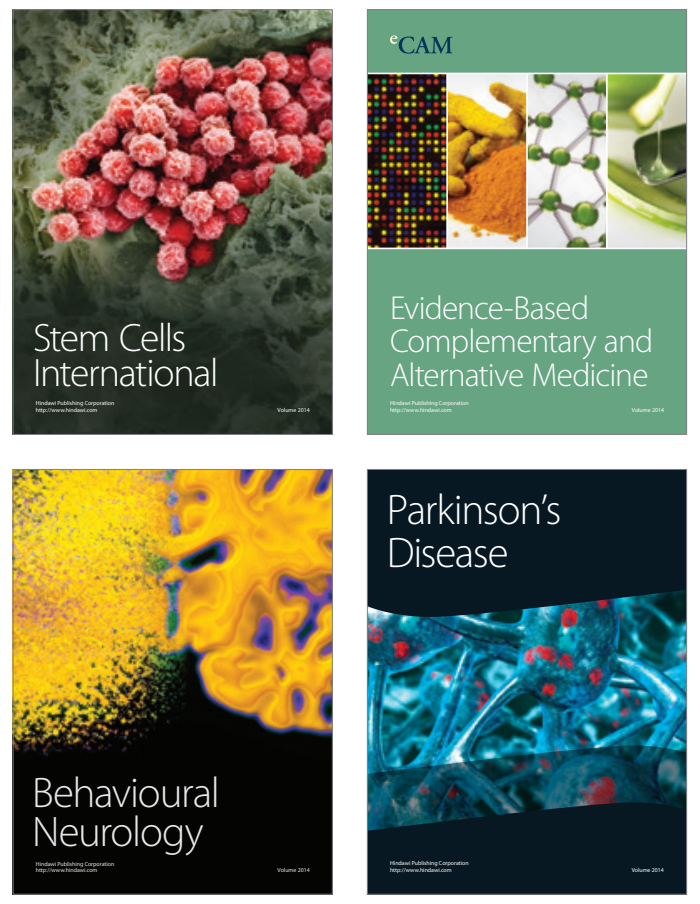
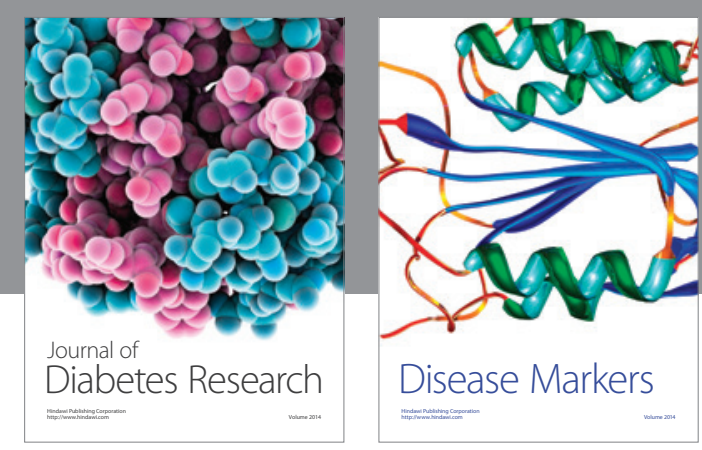

Disease Markers
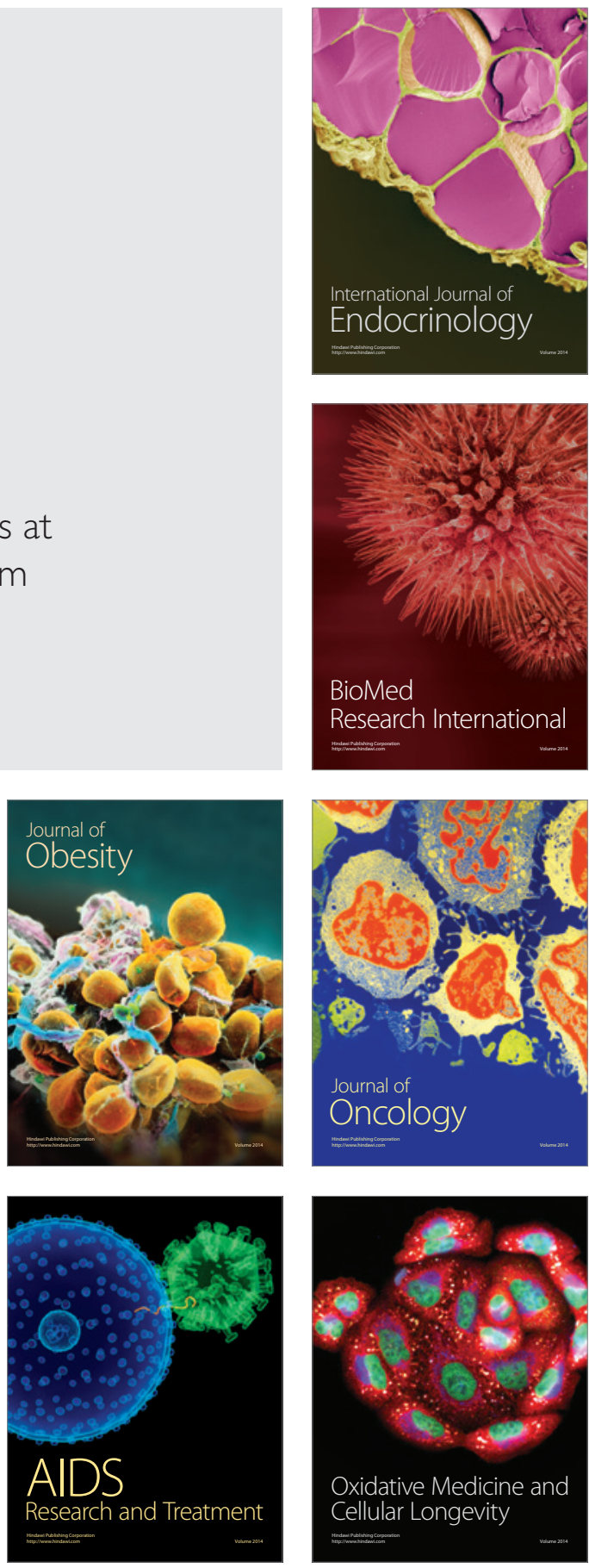